\title{
Unintegrated Gluon Densities from Hadronic Wilson Loops
}

Igor O. Cherednikov*

SCK-CEN, Boeretang 2000, B-2400 Mol, Belgium

Universiteit Antwerpen, Groenenborgerlaan 171, B-2020, Antwerpen, Belgium

E-mail: igor.cherednikov@uantwerpen.be

We briefly overview several operator definitions of gauge-invariant unintegrated (transverse momentum dependent) gluon density function available in the literature, with emphasis on the structure of the Wilson lines or loops. We propose a gauge-invariant formulation of a generating function with maximal path-dependence, which, as distinct from the other approaches, includes an arbitrary Wilson loop, not necessary containing light-like segments, and with no reference to any factorization scheme. After suitable area differentiation, this object can be associated with fully unintegrated gluon distribution function, which allows to apply this approach in lattice simulations.

QCD Evolution 2016

May 30-June 03, 2016

National Institute for Subatomic Physics (Nikhef), Amsterdam

${ }^{*}$ Speaker. 


\section{Unintegrated gluon density functions in the study of high-energy hadronic processes}

The strong interest in transverse-momentum dependent (TMD) parton distribution functions is brought about by the rapid theoretical and experimental development and impressive recent results achieved in the study of the three-dimensional structure of the nucleon, which suggests that not only the longitudinal fraction of the struck parton momenta, normally associated with the Bjorken$x$ variable, but also the two transversal components $\vec{k}_{\perp}=\left(k_{x}, k_{y}\right)$ are taken into account. Experimental programs dealing with high-energy semi-inclusive reactions with polarized and unpolarized hadrons, where the transverse motion and the spin-orbit correlations of the partons are directly accessible, call for an appropriate development of the theory. In well-understood inclusive processes, such as deep-inelastic $e p$-scattering (DIS) or electron-positrion annihilation to hadrons, where no more than one hadron is identified in the initial state, the so-called collinear QCD factorisation approach is applicable. The latter suggests that the longitudinal (parallel to a large light-like momentum in a suitable system) momenta of the patrons are intrinsic (non-perturbative), while their transverse momenta can be created by perturbative radiation effects (parton showers). In less inclusive processes, such as the Drell-Yan lepton pair production, semi-inclusive DIS, hadron-hadron annihilation to jets, Higgs and heavy-flavour production, where two or more hadrons in the initial or final state are detected, one is tempted to go beyond the collinear approximation [1]. The reason is that now the momenta of the particles participating and detected in the process entail a non-planar kinematical setting, which makes it natural to keep not only the collinear but also the transverse components of the parton momenta unintegrated. The so-called transverse-momentum dependent, TMD factorization framework is considered as a unifying QCD-based framework with both mechanisms of the transverse-momentum creation taken into account, that is intrinsic (essentially non-perturbative) as well as the perturbative radiation in parton showers [2].

In practice, factorization theorems provide an important computation tool for the QCD-based study of the intrinsic structure of the nucleon visible in high-energy processes. The TMD or $\mathbf{k}_{\perp^{-}}$ dependent approach to the factorization beyond the collinear approximation $[3,4,5]$ enables the description of both collinear and transverse momenta, as well as polarizations of the quarks and gluons in terms of the TMD parton distribution functions (TMD pdfs). This approach is particularly helpful in the calculation of the differential cross-section in such semi-inclusive processes as the Drell-Yan process and the semi-inclusive deep inelastic scattering $[6,7]$.

At the LHC, the TMD pdfs are suitable for the low- $\mathbf{q}_{T}$ heavy particle production and for the high-energy (small- $x$ ) factorization [2]. Consider, for instance, the Higgs boson production through the gluon-gluon fusion. The effective Lagrangian is given by $[8,9,10]$

$$
L_{\mathrm{eff}}=-\frac{1}{4} g_{\phi} \Phi \mathscr{F}^{\mu \nu} \mathscr{F}_{\mu \nu}
$$

where $g_{\phi}$ stands for the effective coupling, $\Phi$ is the scalar boson field, and $\mathscr{F}^{\mu v}$ is the gluon field strength. The main contribution to the effective theory comes from the top quark loop as the masses of the other quarks are negligible compared to the mass of the top quark. For a recent discussion of the NNLO corrections within the effective theory, see, e.g., Ref. [11]. Hence, the Higgs production via the gluon-gluon fusion naturally introduces the gluon TMD pdfs (gTMDs), given that the gluons 
dominate at high energies in colliders such as the LHC. Presently several approaches to define the gTMD exist in the literature, each being formulated within certain regularization framework to get rid of the specific rapidity (or light-cone) divergences ${ }^{1}$ :

- If we don't make any assumptions about the magnitude of the Bjorken- $x$ and, therefore, cannot use the $\ln x$ as a rapidity cutoff, the "natural" gTMD definition [3, 13] must be supplied with an extra rapidity regulator $\eta$

$$
\begin{aligned}
& \mathbf{G}_{\text {natural }}^{i j}\left(x, \mathbf{k}_{\perp} ; \eta ; h\right) \\
& \sim \int d z^{-} d^{2} z_{\perp} \mathrm{e}^{-i x P^{+} z^{-}+i k_{\perp} z_{\perp}}\left\langle h\left|\mathscr{F}^{i l}(z) \mathscr{W}_{\mathrm{LC}}^{\dagger}(z) \mathscr{W}_{\mathrm{LC}}(0) \mathscr{F}^{l j}(0)\right| h\right\rangle \\
& =\int d z^{-} d^{2} z_{\perp} \mathrm{e}^{-i x P^{+} z^{-}+i k_{\perp} z_{\perp}}\left\langle h\left|\mathscr{F}^{i l}(z) \mathscr{W}_{\mathrm{LC}}^{\text {joint }}(z, 0) \mathscr{F}^{l j}(0)\right| h\right\rangle,
\end{aligned}
$$

where $\mathscr{W}_{\mathrm{LC}}$ stands for a system of Wilson lines with light-like parts (see below). We assume that the two subsystems of the Wilson lines can combine to a 'joint' one:

$$
\mathscr{W}^{\dagger} \mathrm{LC}(z) \mathscr{W}_{\mathrm{LC}}(0)=\mathscr{W}_{\mathrm{LC}}^{\text {joint }}(z, 0)
$$

- In the small- $x$ approximation one can neglect the $x$-dependent contribution in the exponential

$$
\exp \left[i x P^{+} z^{-}\right] \approx 1
$$

so that the $\ln x$ provides a natural rapidity cutoff and the gTMD can be written as [14]

$$
\begin{aligned}
& \mathbf{G}_{\text {small-x }}^{i j}\left(x, \mathbf{k}_{\perp} ; \ln x ; h\right) \\
& \sim \int d z^{-} d^{2} z_{\perp} \mathrm{e}^{i k_{\perp} z_{\perp}}\left\langle h\left|D^{i} \mathscr{W}_{\mathrm{LC}}(z) \mathscr{W}_{\mathrm{LC}}^{\dagger}(z) D^{j} \mathscr{W}_{\mathrm{LC}}(0) \mathscr{W}_{\mathrm{LC}}^{\dagger}(0)\right| h\right\rangle \\
& =\int d z^{-} d^{2} z_{\perp} \mathrm{e}^{i k_{\perp} z_{\perp}}\left\langle h\left|\mathscr{F}^{i l}(z) \mathscr{W}_{\mathrm{LC}}^{\text {joint }}(z, 0) \mathscr{F}^{l j}(0)\right| h\right\rangle,
\end{aligned}
$$

where $z=\left(0^{+}, z^{-}, \mathbf{z}_{\perp}\right)$ and $D^{i}$ is a sort of the covariant derivative with certain boundary conditions.

- Another way to regulate the rapidity singularites is to shift the Wilson lines from the light cone. To this end one adopts a new regulator, $\zeta=(2 v \cdot P) / v^{2}$, where $v$ stands for a non-lightlike vector $[10,15]$

$$
\begin{aligned}
& \mathbf{G}_{\mathrm{off}-\mathrm{LC}}^{i j}\left(x, \mathbf{k}_{\perp}, \zeta ; h\right) \sim \int d z^{-} d^{2} z_{\perp} \mathrm{e}^{-i x P^{+} z^{-}+i k_{\perp} z_{\perp}} \\
& =\int d z^{-} d^{2} z_{\perp} \mathrm{e}^{-i x P^{+} z^{-}+i k_{\perp} z_{\perp}}\left\langle h\left|\mathscr{F}^{i l}(z) \mathscr{W}_{\zeta}^{\text {joint }}(z, 0) \mathscr{F}^{l j}(0)\right| h\right\rangle .
\end{aligned}
$$

\footnotetext{
${ }^{1}$ More detailed analysis of the singularities entails a more sophisticated approach to the TMD pdf definition with a system of the Wilson lines and the soft factors [4, 12].
} 
- Recently another approach has been developed in Refs. [16], which makes use of the hadronic matrix element a Wilson loop operator

$$
\Gamma_{0}(k ; h) \sim \int d^{4} z \mathrm{e}^{-i k z}\left\langle h\left|\mathscr{W}_{\mathrm{C}}\right| h\right\rangle,
$$

where the non-trivial path $\mathrm{C}$ can be deduced from the analysis of colour flows in the factorization formula [21].

Numerous technical details related to the regularization of the rapidity/light-cone singularities, the structure of the Wilson lines which save gauge-invariance for the TMD pdfs, their geometrical layout, universality and breakdown on universality, relation to the colour flows, etc. are discussed, in, e.g., Refs. [4, 18, 19, 20, 21, 22].

Whatever definition of gTMD is adopted, its quantum numbers must correspond to those of the generating matrix element defined in the coordinate space:

$$
\mathbf{G}_{\text {gen. }}^{\mu v \mid \mu^{\prime} v^{\prime}}(z ; h)=\left\langle h\left|\mathscr{F}^{\mu v}(z) \mathscr{W}_{\gamma}(z, 0) \mathscr{F}^{\mu^{\prime} v^{\prime}}(0)\right| h\right\rangle,
$$

where $\mathscr{W}_{\gamma}$ is a generic Wilson line (gauge link) evaluated along a path $\gamma^{2}$.

Note that the evolution equations of the gTMDs differs from those ones of the parton distributions in the collinear case due to the dependence on rapidity regulators. The evolution of the TMDs is determined by the Collins-Soper evolution equation, which is a generalization of the renormalization group equation that governs the evolution of parton densities in the collinear case (for a recent discussion, see Ref. [12] and Refs. therein).

\section{Generating matrix element for fully-unintegrated gluon density}

Following the generally accepted procedure to obtain an operator definition of unintegrated pdfs one starts from the formulation of relevant factorisation framework in a convenient gauge. This obviously yields a gauge-dependent object. Then, through the resummation of gluon corrections in an appropriate approximation one gets a pdf where gauge-invariance is restored by a system of the Wilson lines. Their geometrical situation is uniquely determined by the hard part of a given factorized cross-section, which affects the structure of the gauge links via the relevant colour flows.

An alternative approach starts from a very general function, which is associated to a given unintegrated pdf via the relevant quantum numbers [17]. Such a generic gauge-invariant and strongly path-dependent function is supposed to obey certain evolution equations, defined in the coordinate space, which make it possible to adjust the geometrical structure to a specific factorisation scheme. Therefore, we end up with a gauge-invariant pdf with the Wilson lines, whose path-dependence is prescribed by the factorisation, but the path-dependence comes in a reversed order as compared with the standard approach.

Let us define the operator-valued Wilson exponential evaluated along a given trajectory $\gamma$ from a point $y$ to a point $x$ as

$$
\mathscr{W}_{\gamma}[x, y]=\mathscr{P} \exp \left[i g \int_{y}^{x} d \zeta_{\mu} \hat{A}^{\mu}(\zeta)\right]_{\gamma} .
$$

\footnotetext{
${ }^{2}$ The presence of the gauge links not only ensures the gauge invariance of the gTMDs, but is also a result of the resummation of soft gluons [23].
} 
It obeys the differential equation

$$
\partial_{\mu} \mathscr{W}[x, y]=i g \hat{A}_{\mu}(x) \mathscr{W}[x, y],
$$

which doesn't suggest any changes in the underlying contour $\gamma$. A Wilson line (a loop for closed paths) can be introduced as a matrix element of the operator (2.1) in a vacuum $|v a c\rangle$ or a hadronic $|h\rangle$ state. We are interested now in the laws which govern the behaviour of such matrix elements under variations of the form of the contours. It is known that the shape variations of arbitrary Wilson loops in vacuum can be consistently formulated in terms of the equations of motion in the loop space, namely the Makeenko-Migdal loop equations, Refs. [24]:

$$
\delta_{v}^{\text {path }}(x) \delta_{\mu \nu}^{\text {area }}(x)\left\langle v a c\left|\mathscr{W}_{\gamma}^{1}\right| v a c\right\rangle=N_{c} g^{2} \oint_{\gamma} d z_{\mu} \delta^{(4)}(x-z)\left\langle v a c\left|\mathscr{W}_{\gamma_{x z} \gamma_{z x}}^{2}\right| v a c\right\rangle,
$$

where $\mathscr{W}^{n}$ stands for the $n$-th order product of Wilson exponentials [24]. The so-called path $\delta_{v}^{\text {path }}$ and area $\delta_{\mu \nu}^{\text {area }}$ differential operators are introduced in the loop space to formally define the shape variations of contours. These operators can also be used to formulate the generating function for fully-unintegrated gluon pdfs.

More specifically, making use of non-Abelian Stokes' theorem

$$
\mathscr{W}_{\gamma}=\mathscr{P}_{\gamma} \exp \left[\oint_{\gamma} d \zeta_{\rho} \hat{A}^{\rho}(\zeta)\right]=\mathscr{P}_{\gamma} \mathscr{P}_{\sigma} \exp \left[\int_{\sigma} d \sigma_{\rho \rho^{\prime}}(\zeta) \mathscr{F} \rho \rho^{\prime}(\zeta)\right]
$$

where $\mathscr{P}_{\sigma}$ denotes the area-ordering, and the Mandelstam formula

$$
\begin{aligned}
\delta_{\mu \nu}^{\text {area }}(x) \mathscr{W}_{\gamma} & =\frac{\delta}{\delta \sigma_{\mu \nu}(x)} \mathscr{P}_{\gamma} \exp \left[\oint_{\gamma} d \zeta_{\rho} \hat{A}^{\rho}(\zeta)\right] \\
& =\mathscr{P}_{\gamma} \mathscr{F}^{\mu v}(x) \exp \left[\oint_{\gamma} d \zeta_{\rho} \hat{A}^{\rho}(\zeta)\right]
\end{aligned}
$$

we see that the generating matrix element (1.7) can be re-written as

$$
\begin{aligned}
\tilde{\mathbf{G}}_{\text {gen. }}^{\mu v \mid \mu^{\prime} v^{\prime}}(z ; h) & =\delta_{\mu \nu}^{\text {area }}(z) \delta_{\mu^{\prime} v^{\prime}}^{\text {area }}(0)\left\langle h\left|\mathscr{W}_{\gamma^{[z, 0]}}\right| h\right\rangle \\
& =\delta_{\mu \nu}^{\text {area }}(z) \delta_{\mu^{\prime} v^{\prime}}^{\text {area }}(0) \sum_{X}\left\langle h\left|\mathscr{W}_{\gamma^{[z]}}^{\prime}\right| X\right\rangle\left\langle X\left|\mathscr{W}_{\gamma^{[0]}}^{\prime}\right| h\right\rangle
\end{aligned}
$$

where the underlying (arbitrary) path $\gamma^{[z, 0]}$ must contain the points $z$ and 0 . The key feature of this approach is that one first calculates the generating matrix element $\left\langle h\left|\mathscr{W}_{\gamma^{z z, 0]}}\right| h\right\rangle$, choosing its underlying integration path to fit a specific factorization framework.

Let us demonstrate how this approach works in practice by considering the Abelian gauge theory, where the Wilson loops exponentiate

$$
\begin{aligned}
\left\langle h\left|\mathscr{W}_{\gamma}\right| h\right\rangle & =\left\langle h\left|\mathscr{P}_{\gamma} \exp \left[\oint_{\gamma} d \zeta_{\rho} A^{\rho}(\zeta)\right]\right| h\right\rangle \\
& =\exp \left[-\frac{g^{2}}{2} \oint_{\gamma} d \zeta_{\rho} \oint_{\gamma} d \zeta_{\rho^{\prime}}^{\prime} D^{\rho \rho^{\prime}}\left(\zeta-\zeta^{\prime}\right)\right]
\end{aligned}
$$

The hadronic correlator

$$
D^{\rho \rho^{\prime}}\left(\zeta-\zeta^{\prime}\right)=\left\langle h\left|\mathscr{P}_{\gamma} A^{\rho}(\zeta) A^{\rho^{\prime}}\left(\zeta^{\prime}\right)\right| h\right\rangle
$$


can be parameterized as

$$
D_{\rho \rho^{\prime}}(z)=g_{\rho \rho^{\prime}} D_{1}(z, P)+\partial_{\rho} \partial_{\rho^{\prime}} D_{2}(z, P)+\left\{P_{\rho} \partial_{\rho^{\prime}}\right\} D_{3}(z, P)+P_{\rho} P_{\rho^{\prime}} D_{4}(z, P) .
$$

Hence the area derivative can be calculated straightforwardly

$$
\begin{aligned}
& \frac{\delta}{\delta \sigma_{\mu v}(z)}\left\langle h\left|\mathscr{W}_{\gamma}\right| h\right\rangle=-\frac{g^{2}}{2}\left[\frac{\delta}{\delta \sigma_{\mu v}(z)} \oint_{\gamma} d \zeta_{\rho} \oint_{\gamma} d \zeta_{\rho^{\prime}}^{\prime} D_{\rho \rho^{\prime}}\left(\zeta-\zeta^{\prime}\right)\right]\left\langle h\left|\mathscr{W}_{\gamma}\right| h\right\rangle \\
& =-\frac{g^{2}}{2}\left[\partial_{\mu} \oint_{\gamma} d \zeta_{\rho^{\prime}}^{\prime} D_{v \rho^{\prime}}\left(z-\zeta^{\prime}\right)-\partial_{\nu} \oint_{\gamma} d \zeta_{\rho^{\prime}}^{\prime} D_{\mu \rho^{\prime}}\left(z-\zeta^{\prime}\right)\right]\left\langle h\left|\mathscr{W}_{\gamma}\right| h\right\rangle,
\end{aligned}
$$

and expressed in terms of the invariant functions $D_{1,2,3,4}$. Eq. (2.10) determines, in fact, the shape variations of the hadronic Wilson loops, as distinct from the Makeenko-Migdal equations, which deal with the vacuum Wilson loops. By means of this equation, one can manipulate with the structure of the underlying contours to make them suitable for a given factorization scheme.

To conclude, we have shown that

- Fully unintegrated gluon distribution function can be defined within a completely gaugeinvariant and path-dependent framework based on the loop space formalism in the coordinate representation;

- This approach goes the other way round with respect to the standard one: we start from a gauge-invariant generating function, and then obtains a gluon unintegrated pdf, which is, at least, in principle, adjustable to any specific factorisation scheme;

- The main ingredients of this approach are the hadronic matrix elements of Wilson loops $\left\langle h\left|\mathscr{W}_{\gamma}\right| h\right\rangle$, calculable on the lattice given that the underlying contours $\gamma$ can be chosen in the most convenient way.

\section{References}

[1] J. C. Collins, D. E. Soper and G. F. Sterman, “Factorization of Hard Processes in QCD," Adv. Ser. Direct. High Energy Phys. 5 (1989) 1

[2] R. Angeles-Martinez et al. "Transverse Momentum Dependent (TMD) Parton Distribution Functions: Status and Prospects,” Acta Phys. Polon. B 46 (2015) 2501

[3] J. C. Collins and D. E. Soper, “Back-To-Back Jets in QCD,” Nucl. Phys. B 193 (1981) 381 [Nucl. Phys. B 213 (1983) 545]; "Parton Distribution and Decay Functions,” Nucl. Phys. B 194 (1982) 445; J. C. Collins, "What exactly is a parton density?," Acta Phys. Polon. B 34 (2003) 3103

[4] J. Collins, "Foundations of Perturbative QCD," Cambridge University Press (2011)

[5] D. Boer et al., "Gluons and the quark sea at high energies: Distributions, polarization, tomography," arXiv:1108.1713 [nucl-th]

[6] J. C. Collins, D. E. Soper and G. Sterman, "Transverse Momentum Distribution in Drell-Yan Pair and W and Z Boson Production,” Nucl. Phys. B 250 (1985) 199

[7] P. Nadolsky, D. R. Stump and C. P. Yuan, "Semi-Inclusive Hadron Production at HERA: the Effect of QCD Gluon Resummation,” Phys. Rev. D 61 (2000) 014003 
[8] S. Dawson, “QCD Corrections to Higgs Boson Production: Nonleading Terms in the Heavy Quark Limit,” Nucl. Phys. B 359 (1991) 283

[9] A. Djouadi, M. Spira and P. M. Zerwas, "Production of Higgs Bosons in Proton Colliders. QCD Corrections,” Phys. Lett. B 264 (1991) 440

[10] X. Ji, J.-P. Ma and F. Yuan, “Transverse-Momentum-Dependent Gluon Distributions and Semi-inclusive Processes at Hadron Colliders,” JHEP 2005 (2005) 07

[11] C. Anastasiou and K. Melnikov, "Higgs Boson Production at Hadron Colliders in NNLO QCD," Nucl. Phys. B 646 (2002) 220

[12] T. C. Rogers, “An Overview of Transverse Momentum Depenedent Factorization and Evolution,” Eur. Phys. J. A 52 (2016) no.6, 153

[13] P.J. Mulders and J. Rodrigues, "Transverse Momentum Dependence in Gluon Distribution and Fragmentation Functions,” Phys. Rev. D 63 (2001) 094021

[14] F. Dominguez, C. Marquet, B. W. Xiao and F. Yuan, "Universality of Unintegrated Gluon Distributions at small x,” Phys. Rev. D 83 (2011) 105005

[15] P. Sun, B.-W. Xiao, F. Yuan, “Gluon Distribution functions and Higgs Boson Production at Moderate Transverse Momentum,” Phys. Rev. D 84 (2011) 094005

[16] D. Boer, S. Cotogno, T. van Daal, P. J. Mulders, A. Signori and Y. J. Zhou, "Gluon and Wilson loop TMDs for hadrons of spin $\leq$ 1,” JHEP 1610 (2016) 013; D. Boer, M. G. Echevarria, P. Mulders and J. Zhou, “Single spin asymmetries from a single Wilson loop,” Phys. Rev. Lett. 116 (2016) no.12, 122001; T. van Daal, "Gluon TMDs in the small-x limit,” arXiv:1610.08273 [hep-ph]

[17] I. O. Cherednikov, “Gauge-Invariant Gluon TMD and Evolution in the Coordinate Space,” PoS QCDEV 2015 (2015) 046; “Fully gauge-invariant maximally path-dependent gluon TMD: Coordinate representation,” J. Phys. Conf. Ser. 678 (2016) no.1, 012049

[18] I. O. Cherednikov and N. G. Stefanis, "Renormalization, Wilson lines, and transverse-momentum dependent parton distribution functions,” Phys. Rev. D 77 (2008) 094001; “Wilson lines and transverse-momentum dependent parton distribution functions: A Renormalization-group analysis," Nucl. Phys. B 802 (2008) 146; “Renormalization-group properties of transverse-momentum dependent parton distribution functions in the light-cone gauge with the Mandelstam-Leibbrandt prescription,” Phys. Rev. D 80 (2009) 054008; I. O. Cherednikov, A. I. Karanikas and N. G. Stefanis, "Wilson lines in transverse-momentum dependent parton distribution functions with spin degrees of freedom,” Nucl. Phys. B 840 (2010) 379

[19] A. Idilbi and I. Scimemi, "Singular and Regular Gauges in Soft Collinear Effective Theory: The Introduction of the New Wilson Line T,” Phys. Lett. B 695 (2011) 463; M. G. Echevarria, A. Idilbi and I. Scimemi, "Soft and Collinear Factorization and Transverse Momentum Dependent Parton Distribution Functions,” Phys. Lett. B 726 (2013) 795; M. G. Echevarria, I. Scimemi and A. Vladimirov, “Universal transverse momentum dependent soft function at NNLO,” Phys. Rev. D 93 (2016) no.5, 054004

[20] I. Balitsky and A. Tarasov, "Rapidity evolution of gluon TMD from low to moderate x,” JHEP 1510 (2015) 017; "Gluon TMD in particle production from low to moderate x,” JHEP 1606 (2016) 164

[21] C. J. Bomhof, P. J. Mulders and F. Pijlman, "The Construction of gauge-links in arbitrary hard processes,” Eur. Phys. J. C 47 (2006) 147; C. J. Bomhof and P. J. Mulders, “Non-universality of transverse momentum dependent parton distribution functions,” Nucl. Phys. B 795 (2008) 409 
[22] A. V. Belitsky, X. Ji and F. Yuan, "Final state interactions and gauge invariant parton distributions," Nucl. Phys. B 656 (2003) 165; D. Boer, P. J. Mulders and F. Pijlman, "Universality of T odd effects in single spin and azimuthal asymmetries,” Nucl. Phys. B 667 (2003) 201; A. V. Belitsky and A. V. Radyushkin, "Unraveling hadron structure with generalized parton distributions," Phys. Rept. 418 (2005) 1; X. d. Ji, J. p. Ma and F. Yuan, “QCD factorization for semi-inclusive deep-inelastic scattering at low transverse momentum,” Phys. Rev. D 71 (2005) 034005; X. d. Ji, J. P. Ma and F. Yuan, "Transverse-momentum-dependent gluon distributions and semi-inclusive processes at hadron colliders,” JHEP 0507 (2005) 020;

[23] I. O. Cherednikov, T. Mertens, F. F. Van der Veken, “Wilson Lines in Quantum Field Theory,” De Gruyter, Berlin 2014; I. O. Cherednikov, F. F. Van der Veken, “Parton Densities in QCD,” De Gruyter, Berlin 2016

[24] Y. M. Makeenko and A. A. Migdal, “Exact Equation for the Loop Average in Multicolor QCD,” Phys. Lett. B 88 (1979) 135, [Erratum-ibid. B 89 (1980) 437]; “Quantum Chromodynamics as Dynamics of Loops,” Nucl. Phys. B 188 (1981) 269; [Sov. J. Nucl. Phys. 32 (1980) 431]; Y. Makeenko, “Methods of Contemporary Gauge Theory”, Cambridge University Press, 2002; R. A. Brandt, F. Neri and M. A. Sato, “Renormalization of Loop Functions for All Loops,” Phys. Rev. D 24 (1981) 879; R. A. Brandt, A. Gocksch, M. A. Sato and F. Neri, “Loop Space,” Phys. Rev. D 26 (1982) 3611; J. N. Tavares, “Chen Integrals, Generalized Loops and Loop Calculus,” Int. J. Mod. Phys. A 9 (1994) 4511 